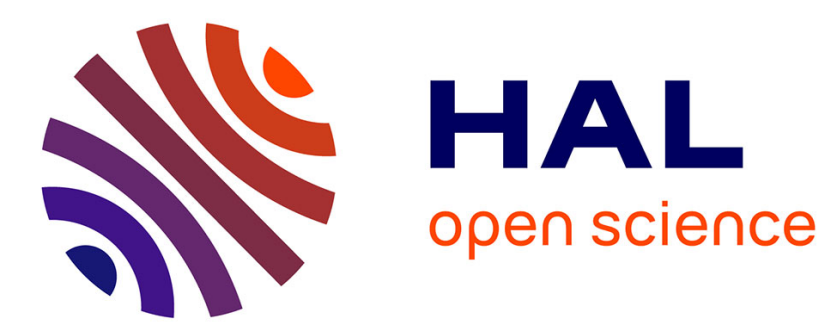

\title{
Points de vue sur les échanges électroniques et leurs usages en formation à distance
}

Christian Depover, Bruno de Lièvre, Gaëtan Temperman

\section{To cite this version:}

Christian Depover, Bruno de Lièvre, Gaëtan Temperman. Points de vue sur les échanges électroniques et leurs usages en formation à distance. STICEF (Sciences et Technologies de l'Information et de la Communication pour l'Éducation et la Formation), 2006, 13, 11 p. hal-00696424

\section{HAL Id: hal-00696424 \\ https://hal.science/hal-00696424}

Submitted on 11 May 2012

HAL is a multi-disciplinary open access archive for the deposit and dissemination of scientific research documents, whether they are published or not. The documents may come from teaching and research institutions in France or abroad, or from public or private research centers.
L'archive ouverte pluridisciplinaire HAL, est destinée au dépôt et à la diffusion de documents scientifiques de niveau recherche, publiés ou non, émanant des établissements d'enseignement et de recherche français ou étrangers, des laboratoires publics ou privés. 


\section{Points de vue sur les échanges électroniques et leurs usages en formation à distance}

Christian DEPOVER, Bruno DE LIÈVRE, Gaëtan TEMPERMAN [Université de Mons-Hainaut, Belgique]

- RÉSUMÉ : Cette contribution, qui résulte de notre participation au symposium Symfonic, se structure autour de quatre questions qui concernent différents aspects liés à l'usage des échanges électroniques en formation à distance. Parmi les aspects discutés à travers ces questions, citons en ordre principal : les modalités de prise en compte des échanges à distance dans le processus de design pédagogique, les modèles susceptibles d'aider à comprendre le rôle pédagogique des échanges électroniques, les complémentarités envisageables entre les différents outils d'échanges, les modalités d'action des échanges électroniques sur l'apprentissage.

MOTS CLÉS : Formation à distance, communication électronique, forum, Chat, modèles d'apprentissage

ABSTRACT : This contribution, which results from our participation in the Symfonic symposium, is structured around four questions which concern different aspects of the use of electronic exchanges in distance learning. Among these aspects, let us name: the modalities of remote exchanges in the pedagogical design process, the models useful to understand the pedagogical role of electronic exchanges, the complementarity between the different tools of exchanges, the effects of electronic exchanges on learning.

KEYWORDS : Distance training, electronic communication, Chat, electronic forum, learning models
1. Introduction
2. Quelle est la place des échanges électroniques dans un dispositif de formation à distance ?
3. Les dispositifs d'échange électronique permettent-ils d'apprendre et comment?
4. Échanges synchrones, quasi synchrones et asynchrones, quelles spécificités et quelles complémentarités?
5. Les forums contribuent-ils à structurer la pensée des individus qui les utilisent ?
6. BIBLIOGRAPHIE 


\section{Introduction}

Dans le prolongement du symposium Symfonic qui s'est tenu à Amiens en janvier 2005, nous avons choisi d'organiser cette contribution sous la forme d'une réflexion à propos de quelques enjeux liés à l'usage des échanges électroniques dans un dispositif de formation à distance. Pour structurer cette réflexion, nous avons utilisé le mode du questionnement en organisant notre travail autour des quatre questions qui sont proposées ci-après.

\section{Quelle est la place des échanges électroniques dans un dispositif de formation à distance?}

Le design pédagogique appliqué à la conception de dispositifs supportés par les TIC s'est longtemps cantonné à prévoir et à organiser des interactions en circuit fermé. Les choses ont fortement changé à ce niveau avec l'arrivée des technologies à distance qui ont donné une place centrale aux interactions avec le tuteur, les pairs et d'autres intervenants externes (tuteurs, modérateurs, gestionnaires de formation...). On est ainsi passé d'une gestion des interactions à circuit fermé à une ouverture très large qui a conduit à remettre en cause les principes de design mobilisés jusqu'alors.

En effet, dans un dispositif fermé, la plupart des interactions sont prévisibles et peuvent donc être gérées par avance. L'intervention directe d'opérateurs humains exige, par contre, une plus grande souplesse dans le design des environnements d'apprentissage qui sont proposés.

Qu'il s'agisse d'apprentissages individuels tutorés ou d'apprentissages en groupe, la création d'un environnement ouvert qui supporte le développement de compétences spécifiques soulève des problèmes nouveaux qui n'ont généralement pas été pris en compte par les procédures de design classiques.

En effet, alors que la présence d'outils supportant les échanges électroniques va aujourd'hui quasiment de soi dans un environnement d'apprentissage, il existe très peu d'éléments sur lesquels on puisse se fonder pour concevoir de tels environnements en se donnant toutes les chances d'assurer leur efficacité pédagogique. A ce propos, Gros (Gros, 2001) souligne la nécessité de définir de nouveaux paradigmes pour le design pédagogique qui prennent en compte les spécificités du tutorat à distance et du travail collaboratif. En particulier, il s'agit, selon cet auteur, de dépasser le paradigme classique en matière de design qui est centré sur les résultats d'apprentissage pour s'intéresser davantage à la manière dont on va pouvoir supporter, encourager, structurer les interactions pour enrichir le processus d'apprentissage.

Dans cette perspective, certains modèles de structuration des environnements d'apprentissage à distance ont été proposés en particulier en ce qui concerne l'écriture de scénarios d'apprentissage en groupe par des auteurs tels que (Dillenbourg, 2002) et (Depover et al., 2003). Dans ces modèles, il s'agit avant tout d'identifier les variables de structuration pertinentes (composition des groupes, modalités d'enchaînement des tâches, modes de suivi, modalités d'interaction...) en vue d'assurer la richesse des interactions à distance.

Malgré leur intérêt, ces modèles de structuration ne fournissent toutefois pas une méthodologie de design au sens habituel du terme. En effet, une telle méthodologie devrait comporter une conceptualisation explicite des liens qui existent entre les objectifs pédagogiques poursuivis et les moyens mis en œuvre pour les atteindre. En d'autres termes, il s'agirait ici d'identifier les modalités d'interaction qu'il conviendrait de privilégier en vue de faciliter le développement de compétences explicitement attendues au terme de la formation. Il s'agit là d'un jalon essentiel sur lequel les chercheurs en matière de dispositifs d'interaction à distance devraient concentrer leur attention.

En l'absence d'une conceptualisation claire de ce que peuvent apporter au processus d'apprentissage des dispositifs d'échange à distance tels que les forums ou les Chat, il n'y a rien d'étonnant de les retrouver 
associés à bon nombre de dispositifs de formation sans que leur pertinence pédagogique soit pour autant avérée. Plutôt que de résulter d'un choix de design, la décision d'associer un dispositif d'échange à distance à un environnement d'apprentissage se justifie le plus souvent par sa facilité de mise en œuvre ou encore par le fait qu'il ne pourra de toute manière pas faire de tort.

Pour reprendre les deux paradigmes proposés par (Gros, 2001), le développement d'une méthodologie de design adaptée aux environnements d'apprentissage à distance devrait permettre de faire converger le paradigme classique centré sur les résultats et le paradigme émergent centré sur le processus en identifiant clairement comment certaines formes d'interactions contribuent au développement de certaines compétences.

Pour caractériser la manière dont les interactions sociales contribuent au développement de certaines compétences, (Doise et Mugny, 1997) parlent de causalité circulaire. Les interactions sociales favorisent le développement de capacités cognitives plus évoluées qui permettent à leur tour des interactions sociales plus complexes.

Développement social et acquisition de compétences cognitives sont donc intimement liés et profitent l'un de l'autre. Comme le soulignait déjà (Vygotsky, 1962), (p. 104) «Ce qu'un enfant peut faire aujourd'hui en collaborant avec autrui, il peut le faire seul demain ». De plus, en affirmant que les interactions inter-individuelles sont à l'origine des coordinations intra-individuelles, cet auteur met clairement en évidence le fondement social de tout apprentissage.

Si nous acceptons cette conception, nous sommes amenés à considérer que les forums constituent un lieu privilégié pour l'apprentissage et qu'il est essentiel que la structuration de l'environnement d'apprentissage reflète cette situation. En particulier, il convient que les forums soient structurés en fonction des compétences visées par le dispositif en mettant clairement en évidence leur apport spécifique mais aussi leur articulation avec d'autres modalités d'interaction ou de mise à disposition d'informations. Il s'agit, comme nous l'avons rappelé ci-avant, de réfléchir davantage en termes de dispositif de formation au sein duquel le forum ne constitue qu'un outil parmi d'autres dont l'efficacité dépendra en grande partie de la manière dont il sera intégré au dispositif dans son ensemble.

\section{Les dispositifs d'échange électronique permettent-ils d'apprendre et comment?}

L'utilisation des forums dans un contexte d'apprentissage à distance peut prendre différentes formes. Parmi celles-ci, les forums basés sur des échanges textuels centrés sur une tâche commune, plus ou moins précise, à réaliser dans un espace temporel défini sont de loin les plus fréquents. Placés dans ce cadre d'interaction, les apprenants sont amenés à s'engager dans des échanges verbaux qui sont censés développer chez eux certaines formes de compétences en particulier à l'occasion d'activités qui les conduisent à élaborer une vision partagée d'un domaine de connaissance (Schwartz, 1995).

Pour comprendre comment un forum permet d'apprendre, il est donc nécessaire de s'interroger sur la manière dont cette compréhension commune peut être construite.

Pour cela, une première distinction nous paraît nécessaire en ce qui concerne la définition de l'espace temporel. Une discussion s'organisant sur quelques jours à propos d'un sujet bien défini n'a souvent rien à voir avec les débats qui peuvent prendre place dans le cadre de véritables communautés d'apprentissage dont les échanges peuvent s'étaler sur plusieurs mois, voire plusieurs années.

Lorsqu'il s'agit d'échanges organisés sur le long terme, ceux-ci s'inscrivent bien souvent dans le contexte d'une culture partagée qui simplifie la communication et favorise certaines formes de compréhension mutuelle. Une discussion ponctuelle organisée pour les besoins d'un cours ou d'une formation bien 
précise comporte souvent un caractère artificiel qui limite l'engagement personnel des participants.

Comme le souligne (Dillenbourg, 2006), la construction d'une vision commune exige que les participants puissent accéder au même espace d'information. Pour cela, il est souvent utile de s'appuyer sur plusieurs moyens de communication à distance disposant chacun de leurs propres spécificités. Ainsi, des outils permettant la communication synchrone comme le Chat pourront apporter un complément utile notamment pour soutenir certains processus de négociation. L'intérêt de supports graphiques comme le «tableau blanc» ou le MOO n'est pas non plus à négliger lorsqu'il s'agit de se représenter l'espace d'activité dans lequel la discussion prendra place.

Bien entendu, il ne suffit pas d'avoir accès aux mêmes informations pour qu'on puisse parler de vision commune. Cette dernière est l'aboutissement d'un processus qui s'appuie sur des informations partagées mais elle se construit au fil des discussions et des débats pour lesquels le forum constitue un lieu privilégié.

(Clark et Brennan, 1991) ont proposé un modèle pour tenter de comprendre comment se construit une compréhension mutuelle (grounding) dans le cadre d'un travail collectif. A cette occasion, ils ont mis en évidence l'importance des éléments permettant de signifier à l'autre que l'on a compris (évidence positive) ou que l'on n'a pas compris son message (évidence négative). Lorsqu'une incompréhension est identifiée, un mécanisme de «réparation » intervient qui permet de réajuster le message en fonction de son destinataire. Ce modèle conduit également à dégager ce que les auteurs appellent le principe d'effort collaboratif minimum qui engendre certaines incompréhensions réparées par des «tours de parole » supplémentaires.

Depuis les travaux de Clark et Brennan qui sont déjà relativement anciens, d'autres perspectives sont apparues pour expliquer les mécanismes en œuvre dans la compréhension mutuelle en se basant notamment sur l'analyse de l'argumentation (Baker, 2003). D'autres auteurs comme (Cherubini et Van Der Pol, 2005) affirment que le niveau d'analyse utilisé dans la plupart des études fait appel à une granularité trop fine pour conduire à une réelle compréhension des mécanismes cognitifs en jeu. Ils mettent notamment en évidence la nécessité, pour prendre en compte les erreurs de compréhension qui apparaissent dans un échange et les réparer, de considérer la perspective de l'autre.

De ces travaux, nous retiendrons l'idée que les échanges dans une situation d'apprentissage collectif reposent sur un principe d'économie qui génère de nombreuses incompréhensions qui doivent être réparées pour pouvoir construire une compréhension commune. Pour construire cette compréhension partagée, il est également important d'élaborer des références communes permettant de prendre en compte la perspective des autres participants et, à terme, de se construire une perspective partagée. De ce point de vue, les forums qui se structurent dans le temps sous la forme d'une communauté devraient s'avérer plus efficaces puisque c'est justement l'un des buts d'une communauté que de se construire une culture partagée.

L'apprentissage dans un forum repose sur une véritable pédagogie de l'erreur ; en effet, c'est à travers les problèmes de communication qui apparaîtront que les partenaires construiront une représentation et une compréhension communes de la situation en élaborant des explications, en se justifiant, en explicitant leurs points de vue... Plutôt que de les considérer comme des phénomènes négatifs perturbant la communication, les difficultés de compréhension constituent au contraire le ferment à partir duquel se construira l'apprentissage. Pour cela, il est important que les situations sur lesquelles porteront les échanges soient suffisamment complexes pour mobiliser les apprenants et pour offrir des occasions de réparer les problèmes de compréhension qui apparaîtront.

Les difficultés de compréhension qui peuvent surgir dans une interaction s'apparentent à la notion de conflit cognitif proposée par les socio-constructivistes comme point de départ à des interactions susceptibles de donner lieu à des apprentissages approfondis. Toutefois, Clark et Brennan (Clark et 
Brennan, 1991) considèrent que, pour qu'un dialogue porteur d'apprentissage se mette en place, il suffit qu'une légère différence dans les points de vue ou encore qu'une incompréhension apparaissent, alors que la notion de conflit fait davantage référence à une opposition franche ou à des divergences profondes liées, par exemple, à des différences de prérequis ou de maturation cognitive.

La gestion des incompréhensions dans une interaction à distance et la prise en charge d'un conflit cognitif peuvent, à notre sens, être utilisées de manière complémentaire pour alimenter les échanges et les débats au sein d'un forum avec pour objectif de dépasser une difficulté passagère mais surtout de construire des compétences de haut niveau. Il ne faut pas perdre de vue que, dans le contexte qui nous occupe, notre intérêt pour les forums se situe dans leur capacité à faire naître des apprentissages significatifs. Dès lors, que ces apprentissages se réalisent à partir d'un conflit induit par le concepteur pédagogique ou sur la base d'une incompréhension surgie spontanément en cours d'échange n'a finalement guère d'importance.

\section{4. Échanges synchrones, quasi synchrones et asynchrones, quelles spécificités et quelles complémentarités?}

Pour le concepteur pédagogique soucieux d'assurer l'efficacité des dispositifs d'apprentissage qu'il propose à ses pupilles, le choix entre échanges synchrones et asynchrones n'est pas toujours aisé.

Dans certains contextes, son pragmatisme l'amènera à privilégier la modalité d'échange la moins contraignante pour les parties impliquées dans les interactions. La possibilité de recevoir les messages en permanence, de les revoir et de les traiter selon ses disponibilités plaide largement en faveur des échanges asynchrones lorsqu'on s'adresse à des apprenants difficiles à réunir au même moment. En particulier, les technologies asynchrones permettent d'engager les apprenants dans des discussions qui se développent dans le temps en libérant les intervenants des contraintes d'agenda qui pèsent sur la discussion synchrone en face à face ou à distance.

Dans la réalité, le choix entre échanges immédiats et différés n'est pas aussi tranché qu'il peut y paraître à première vue. Tout d'abord, beaucoup de dispositifs mettent à la disposition des apprenants les deux possibilités en laissant soit le choix de la modalité d'échange aux utilisateurs soit en leur imposant une modalité pour certaines activités et une autre pour d'autres activités. Ensuite, l'utilisateur d'un dispositif d'échange qualifié d'asynchrone peut bénéficier, s'il dispose d'un équipement informatique performant, de possibilités d'interaction très rapides comparables à celles d'un dispositif synchrone.

Compte tenu du rapprochement des possibilités des dispositifs d'interaction, on peut se demander s'il reste pertinent ou pas d'en faire une variable de design. En d'autres termes, faut-il décider a priori de proposer à l'apprenant l'accès à un Chat plutôt qu'à un forum ou doit-on plutôt lui offrir les deux possibilités en le laissant décider celle à laquelle il fera appel à un moment donné de son apprentissage en fonction des exigences du contexte humain et technologique auquel il est confronté ?

Un certain nombre de résultats de recherche soulignent toutefois la possibilité de tirer parti de certaines formes de complémentarité existant entre dispositifs synchrones et asynchrones.

(Clark et Brennan, 1991) mettent en évidence que certains médias favorisent davantage la construction d'une compréhension commune que d'autres. A ce niveau, ils distinguent huit types de contraintes qui sont susceptibles d'influencer l'efficacité d'un média: coprésence, visibilité, audibilité, co-temporalité, simultanéité, séquentialité, permanence et révision. L'hypothèse avancée par ces auteurs est qu'un média sera d'autant plus performant en matière d'apprentissage collectif qu'il rencontrera ces différentes contraintes.

Par exemple, si l'on compare la conversation en face à face au mail, le face à face rencontrera l'ensemble 
de ces critères à l'exception de la révision. En effet, lorsqu'une parole est échangée, on ne peut plus la corriger avant que le récepteur en prenne connaissance. Par contre, le mail sera jugé moins efficace en fonction de ces contraintes parce qu'il n'assure ni la coprésence ni la visibilité, pas plus que la cotemporalité (les échanges ne sont pas reçus au fur et à mesure qu'ils s'élaborent) ou la simultanéité (plusieurs messages ne peuvent être transmis en même temps comme c'est le cas en face en face lorsque le récepteur hoche la tête pour acquiescer au message pendant qu'il est délivré verbalement).

Lorsque les échanges se déroulent à travers un média pour lequel une ou plusieurs des caractéristiques cidessus sont manquantes, l'apprenant devra compenser cette faiblesse. Par exemple, l'usage d'un média comme le forum pénalisera les échanges notamment par le fait qu'il ne prend pas en charge la simultanéité et la séquentialité. Il conviendra dès lors d'insister pour que le seul canal de communication disponible (l'écrit) soit le plus explicite possible en soignant les formulations ou encore en ajoutant des informations par rapport aux intentions de l'émetteur sous la forme d'émoticons. Une autre solution, pour compenser la faiblesse relative de certains médias, consiste à utiliser en parallèle plusieurs moyens de communication.

L'hypothèse de complémentarité des médias a été testée dans le cadre d'une étude menée par (Depover et De Lièvre, 2005). Cette étude a notamment permis de montrer qu'il existait une forme de compensation d'un mode de communication par rapport à l'autre (Chat versus forum) de sorte que lorsque l'usage de l'un diminue, l'autre augmente et réciproquement. L'analyse des unités de sens a également permis de mettre en évidence le rôle prépondérant de l'argumentation dans les échanges basés sur le Chat alors que celle-ci est moins présente dans les forums. On argumente et on négocie davantage à travers les Chat (445 unités de sens contre 122) et dans des proportions plus importantes $(18,7 \%$ des interventions contre 13,4\%). Par contre, la proportion des unités de sens réservées à la planification de la tâche est plus importante dans les forums $(15,2 \%$ contre $8,4 \%)$ bien, qu'en nombre absolu, l'utilisation des Chats reste prépondérante à ce niveau (139 unités de sens contre 199).

Le lien entre la nature des informations échangées et l'utilisation d'un outil de communication a aussi été souligné dans une étude réalisée par (Dillenbourg et al., 1996) dans laquelle les sujets avaient le choix entre l'utilisation du MOO (un système d'échange synchrone basé sur une métaphore spatiale) et celle d'un tableau blanc (whiteboard). Cette étude a permis de montrer que le choix d'un outil de communication était lié au type d'informations échangées. En effet, les sujets avaient tendance à utiliser le MOO pour échanger des informations de nature transitoire comme des inférences ou des étapes dans un raisonnement et le tableau blanc pour échanger des informations à caractère plus persistant comme des faits.

Bien que les études dans le domaine soient encore en nombre limité, il semblerait qu'il existe bien des spécificités liées aux moyens d'interaction à distance et que les apprenants utilisent ces spécificités en fonction de leurs besoins. A ce propos, (Depover et De Lièvre, 2005) ont notamment montré le rôle que pouvait jouer la pression temporelle dans le fait de préférer le Chat au forum à l'occasion de l'analyse des interactions mises en œuvre dans deux dispositifs de formation à distance portant respectivement sur l'usage des tests psychologiques en ligne (Débat d'opinion) et sur une formation à l'intercompréhension des langues romanes (Galanet).

Comme l'ont mis en évidence à propos des TIC des auteurs comme (Rabardel, 1995) ou (Perriault, 1989), les logiques d'usage conduisent souvent à des formes d'appropriation des technologies fort éloignées de celles auxquelles avaient pensé leurs concepteurs. Il n'y a, à notre sens, pas de raison qu'il en soit autrement des outils d'interaction à distance. Dès lors, au-delà de certaines spécificités associées aux outils qui semblent se dégager, les usages réels seront définis par les utilisateurs qui profiteront de la liberté qui leur est offerte par le dispositif pour gérer les interactions à distance en fonction de la perception de leurs propres besoins et de la connaissance qu'ils ont des outils mis à leur disposition. Dans les mains d'un utilisateur averti, il y a de fortes chances qu'un outil d'interaction dont le potentiel est jugé limité puisse conduire à des interactions riches et porteuses d'apprentissage alors que le même outil sera 
très pénalisant pour un sujet novice dans la pratique des échanges à distance.

\section{Les forums contribuent-ils à structurer la pensée des individus qui les utilisent?}

Certains auteurs comme (Pea, 1985) ou (Salomon, 1993) plaident depuis longtemps en faveur de l'idée que les outils et en particulier les outils technologiques contribuent à façonner la pensée. Plus particulièrement, en ce qui concerne le travail de groupe, (Rambusch et al., 2004) ont montré le rôle crucial des artefacts technologiques dans la coordination des activités collectives. Néanmoins, malgré le nombre croissant d'études qui portent sur les effets des artefacts sur la cognition, nous ne disposons que de très peu d'éléments qui nous permettent de comprendre la manière dont les individus utilisent les artefacts dans le travail pour soutenir leurs activités cognitives.

En ce qui concerne plus particulièrement les forums, il nous paraît légitime de considérer deux sources principales d'action possible sur l'élaboration de la pensée des individus. D'une part, le dispositif technologique qui, par ses caractéristiques matérielles, favorisera certaines activités, incitera l'individu à adopter certains comportements et d'autre part, l'environnement humain qui, par l'intermédiaire du modérateur et des pairs, orientera les débats et infléchira la pensée des individus qui y prendront part.

Pour tenter de cerner la manière dont les caractéristiques matérielles des forums sont susceptibles d'influencer le déroulement de la pensée des utilisateurs, nous nous appuierons sur les critères proposés par (Dix et al., 2004) qui distinguent deux types d'éléments : les trigger (déclencheurs) qui permettent de signaler que vous devez faire quelque chose et les placeholder qui indiquent ce que vous devez faire.

Les trigger servent à attirer l'attention sur différentes activités à réaliser :

- indiquer qu'une tâche doit intervenir après une autre (Envoyez-moi le fichier X dès réception de ce message) ;

- mettre en évidence des actions qui doivent intervenir avec une certaine régularité (Consultez le contenu du forum au moins deux fois par jour) ;

- faire référence à un événement extérieur (Rendez-vous sur le Chat de votre groupe demain à 2 heures GMT).

Les placeholder soulignent ce qui doit être fait :

- en gardant une trace de la progression dans le travail (le modérateur fait un bilan des points qui sont considérés comme acquis par le groupe) ;

- en rappelant la séquence des opérations à réaliser (le modérateur rappelle les points qui restent à traiter) ;

- en structurant les échanges (un participant propose de créer un nouveau fil de discussion) ;

- en rappelant le contexte dans lequel s'inscrivent les échanges.

A partir de ces catégories, on se rend aisément compte que l'artefact technologique mis en œuvre dans les forums contribue assez peu à structurer la réflexion des individus qui y participent alors que les interventions humaines au sein du forum jouent un rôle beaucoup plus important à ce niveau et suppléent en quelque sorte aux faiblesses du dispositif technologique.

Même si certains forums s'efforcent de proposer des éléments susceptibles de favoriser l'organisation des discussions comme c'est le cas lorsqu'ils comportent, par exemple, des outils pour gérer les fils de 
discussion ou des ouvreurs de phrase pour faciliter les dialogues, l'artefact technologique mis en ouvre dans la plupart des forums reste peu structurant.

Faute d'artefact technologique efficace, la pertinence des échanges qui se déroulent au sein d'un forum dépend donc essentiellement des interventions humaines qui y sont mises en œuvre. En ce qui concerne les interactions entre pairs, on sait que certaines formes de régulation peuvent apparaître spontanément en particulier lorsque les groupes sont «modérément hétérogènes » c'est-à-dire lorsqu'ils sont constitués d'un élève moyen et d'un élève fort ou d'un élève moyen avec un élève faible (Webb, 1991).

Dans la pratique toutefois, les régulations spontanées au sein des groupes sont souvent limitées par le faible nombre d'interventions ou par la prégnance des interventions du tuteur. Une étude de (George, 2001) auprès d'étudiants de la télé-université a, par exemple, montré que les relations apprenantsformateurs prédominent très largement sur les relations entre apprenants.

En ce qui concerne les interventions du tuteur qui, comme nous venons de le souligner, occupent souvent une place importante dans le flux d'échanges, il n'est pas toujours possible d'en garantir la qualité. En effet, le tutorat dans un dispositif d'apprentissage à distance constitue une ressource rare et coûteuse. Les personnes qui disposent d'une expertise suffisante par rapport aux contenus d'apprentissage et qui maîtrisent les techniques propres au tutorat à distance sont souvent difficiles à mobiliser en nombre suffisant pour assurer le suivi de cohortes d'étudiants qui, en formation à distance, sont généralement importantes.

Parmi les compétences attendues du tuteur en ligne, les compétences organisationnelles c'est-à-dire celles qui visent à structurer les échanges sont souvent perdues au milieu de bon nombre de compétences d'ordre divers : pédagogiques, techniques, sociales, disciplinaires... Dès lors, rien d'étonnant à ce que les tuteurs n'accordent pas toujours à cette fonction, pourtant essentielle pour l'efficacité et pour la longévité d'un forum (faute de relances efficaces, un forum peut s'éteindre très rapidement), la place qu'il conviendrait.

La question qui se pose à ce niveau est de savoir comment agir pour faire évoluer la situation. Faut-il investir dans l'amélioration du design technologique des forums pour développer leurs possibilités en ce qui concerne la régulation des échanges ou est-il préférable de concentrer ses efforts sur la formation des tuteurs?

Sans négliger l'intérêt d'une amélioration du potentiel structurant des forums et la nécessité de former les tuteurs, une troisième piste nous paraît également intéressante à envisager à ce niveau. Celle-ci porterait sur l'amélioration des outils technologiques mais en se centrant non pas sur la structuration des échanges mais plutôt sur l'information des tuteurs afin de faciliter le suivi des apprenants qu'ils ont en charge. Comme le mettent en évidence Dimitracopoulou et Bruillard dans ce volume, il s'agit là d'un domaine de recherche en plein développement qui a conduit à la production de plusieurs dispositifs permettant d'aider les tuteurs dans leur travail de suivi et de régulation des interactions mises en œuvre dans le cadre de forums de discussion. Dans notre département, un dispositif de ce type est actuellement en cours d'évaluation à partir de la plateforme Esprit ${ }^{1}$. Ce dispositif permet notamment d'afficher un tableau de bord qui informe le tuteur de l'état des interactions dans les différents groupes qu'il gère à un moment donné et qui l'aide à replacer ces échanges dans un cadre plus large (Temperman et al, 2007). Le recours à de tels outils devrait non seulement contribuer à alléger le travail des tuteurs mais aussi, à terme, les amener à améliorer la qualité de leurs interventions en les aidant à prendre en compte le déroulement global des apprentissages.

\section{BIBLIOGRAPHIE}

BAKER, M.J. (2003). Computer-mediated Argumentative interactions for the co-elaboration of scientific notions. In J. Andriessen, M.J. BAKER \& D. SUTHERS (Eds.) Arguing to Learn: Confronting 
Cognitions in Computer-Supported Collaborative Learning environments, p. 47-78. Dordrecht, The Netherlands : Kluwer Academic Publishers.

CHERUBINI M., VAN DER POL, J.(2005). Grounding is not shared undestanding : distinguishing grounding at an utterance and knowledge level. CONTEXT'05, The Fifth International and Interdisciplinary Conference on Modeling and Using Context, Paris, France.

CLARK, H. H., \& BRENNAN, S. E. (1991). Grounding in communication. In L. B. Resnick, J. Levine, \& S. D. Teasley (Eds.), Perspectives on socially shared cognition (pp. 127-149). Washington, DC: APA. Reprinted in R. M. BAECKER (Ed.), Groupware and computer-supported cooperative work: Assisting human-human collaboration (p. 222-233). San Mateo, CA: Morgan Kaufman Publishers, Inc.

DEPOVER C., QUINTIN J-J., DE LIEVRE B. (2003) Un outil de scénarisation de formations à distance basées sur la collaboration. In : DESMOULINS, C, MARQUET, P. \& BOUHINEAU, D. (Eds). Environnements informatiques pour l'apprentissage humain, p. 115-126, Strasbourg, 2003.

DEPOVER, C. \& DE LIèVRE, B. (2005). Analyse des usages des outils de communication médiatisée par ordinateur dans le cadre de deux scénarios de formation à distance. Symposium Symphonic, Amiens : Université de Picardie.

DILLENBOURG P., TRAUM, D. (2006). Sharing solutions : persistence and grounding in multimodal collaborative problem solving. Journal of the Learning sciences, vol. 15, n 1, p. 121-151.

DILLENBOURG, P. (2002). Over-scripting CSCL : The risks of blending collaborative learning with instructional design. In : P.A. Kirschner (Ed.). Three worlds of CSCL : Can we support CSCL ? Den Haag : Open Universiteit.

DILLENBOURG, P., TRAUM, D. \& SCHNEIDER D. (1996). Grouping in multi-modal task oriented collaboration. Proceedings of the European Conference on Artificial Intellingence in Education. 415-425, Lisbon, Portugal.

DIX, A., RAMDUNY-ELLIS,, D. \& WILKINSON, J. (2004). Trigger analysis - understanding broken tasks. In : D. Diaper \& N. Stanton (Eds.), The Handbook of Task Analysis for Human-Computer Interaction. Mahwah : Erlbaum

DOISE, W. \& MUGNY, G. (1997) Psychologie sociale et développement cognitif. Paris : Armand Colin.

GEORGE S. (2001), Apprentissage collectif à distance. SPLACH : un environnement informatique support d'une pédagogie de projet, Thèse en Informatique, Université du Maine.

GROS, B. (2001). Instructional design for computer-supported collaborative learning in primary and secondary education. Computer in Human Behavior, 17, p. 439-451

PEA, R.D. (1985) Beyond amplification : using the computer to reorganize mental functioning. Educational Psychologist, 20(4), p. 167-182.

PERRIAULT J. (1989) La logique de l'usage. Essai sur les machines à communiquer. Paris : Flammarion.

RABARDEL, P. (1995). Les hommes et les technologies, Paris : Armand Colin.

RAMBUSCH, J., SUSI, T. \& ZIEMKE, T. (2004) Artefacts as mediators of distributed social cognition : a case study. In : K. FORBUS, D. GENTNER \& T. REGIER (Eds.), Proceeding of the $26^{\text {th }}$ Annual Conference of the Cognitive Science Society..

SALOMON G. (1993). No distributions without individuals cognition : a dynamic interaction view. In : Salomon G. (Ed.), Distributed cognitions. Cambridge : University Press. 
TEMPERMAN, G., DEPOVER, C. \& DE LIEVRE, B. (2007). Le tableau de bord, un outil d'awareness asynchrone. Analyse de son usage dans un environnement collaboratif à distance. A paraître

SCHWARTZ, D.L. (1995). The emergence of abstract dyad representations in dyad problem solving. The Journal of the Learning Sciences, 4 (3), p. 321-354.

VYGOTSKY, L.S. (1962). Thought and Language. Cambridge : MIT Press.

WEBB, N.M. (1991). Task related verbal interaction and mathematics learning in small groups. Journal for research in mathematics education, 22 (5), p 366-389.

${ }^{1}$ ESPRIT - Environnement Scénarisé pour l'aPRentissage Interactif et Tutoré - Unité de Technologie de

l'Education - Université de Mons-Hainaut et Grenoble Universités - (http://ute2.umh.ac.be/esprit )

\section{A propos des auteurs}

Christian DEPOVER est professeur à l'Université de Mons-Hainaut (Belgique), il enseigne également à l'Université libre de Bruxelles. Il anime un centre de recherche et de développement consacré aux usages des technologies en éducation et au E-learning (Unité de Technologie de l'Éducation). Il partage son temps entre l'enseignement, la recherche et la consultation auprès d'organisations internationales.

Adresse : Université de Mons-Hainaut, 18, place du Parc - 7000 Mons - Belgique

Courriel : Christian.Depover@umh.ac.be

Toile : http://ute.umh.ac.be/site_ute2/

Bruno DE LIÈVRE est chargé de cours à l'Université de Mons-Hainaut, il est responsable du service de Pédagogie Générale et des Médias Educatifs. Les contenus des enseignements qu'il assure et des recherches qu'il mène sont relatifs à la pédagogie et la didactique générale ainsi qu'à l'usage des technologies dans l'éducation et la formation, domaine dans lequel il a réalisé sa thèse de doctorat ainsi que publié de nombreux articles.

Adresse : Université de Mons-Hainaut, 18, place du Parc - 7000 Mons - Belgique

Courriel : Bruno.Delievre@umh.ac.be

Toile : http://ute.umh.ac.be/site_ute2/

Gaëtan Temperman est chercheur au sein de l'Unité de Technologie de l'Éducation à l'Université de Mons-Hainaut. Ses activités de recherche portent sur l'analyse de l'usage des outils dédiés à la collaboration (awareness, forum,...) dans les environnements d'apprentissage à distance.

Adresse : Université de Mons-Hainaut, 18, place du Parc - 7000 Mons - Belgique

Courriel : Gaetan.Temperman@umh.ac.be

Toile : http://ute.umh.ac.be/site_ute2/

\section{Référence de l’article :}

Christian DEPOVER, Bruno DE LIÈVRE, Gaëtan TEMPERMAN, Points de vue sur les échanges électroniques et leurs usages en formation à distance, Revue STICEF, Volume 13, 2006, Rubrique, ISSN : 1764-7223, mis en ligne le 28/02/2007, http://sticef.org

(C) Revue Sciences et Technologies de 1'Information et de la Communication pour l'Éducation et la Formation, 2006 
